Bull. Korean Math. Soc. 52 (2015), No. 5, pp. 1649-1660

http://dx.doi.org/10.4134/BKMS.2015.52.5.1649

\title{
REDUCING SUBSPACES FOR A CLASS OF TOEPLITZ OPERATORS ON THE BERGMAN SPACE OF THE BIDISK
}

\author{
Mohammed Albaseer, Yufeng Lu, and Yanyue Shi
}

ABSTRACT. In this paper, we completely characterize the nontrivial reducing subspaces of the Toeplitz operator $T_{z_{1}^{N} \bar{z}_{2}^{M}}$ on the Bergman space $A^{2}\left(\mathbb{D}^{2}\right)$, where $N$ and $M$ are positive integers.

\section{Introduction}

Let $\mathbb{D}$ be the open unit disk in the complex plane $\mathbb{C}$. For $-1<\alpha<\infty$, let $L^{2}\left(\mathbb{D}, d A_{\alpha}\right)$ be the Hilbert space of square integrable functions on $\mathbb{D}$ with the inner product

$$
\langle f, g\rangle_{\alpha}=\int_{\mathbb{D}} f(z) \overline{g(z)} d A_{\alpha}(z), \quad f, g \in A_{\alpha}^{2}(\mathbb{D})
$$

where

$$
d A_{\alpha}(z)=(\alpha+1)\left(1-|z|^{2}\right)^{\alpha} d A(z),
$$

and $d A$ is the normalized area measure on $\mathbb{D}$.

The weighted Bergman space $A_{\alpha}^{2}(\mathbb{D})$ is the subspace of $L^{2}\left(\mathbb{D}, d A_{\alpha}\right)$ consisting of all the analytic functions in $\mathbb{D}$. We denote

$$
\gamma_{n}=\left\|z^{n}\right\|_{\alpha}=\sqrt{\frac{n ! \Gamma(2+\alpha)}{\Gamma(n+\alpha+2)}}
$$

for $n=0,1,2, \ldots$ Therefore,

$$
\|f\|_{\alpha}^{2}=\sum_{n=0}^{+\infty} \gamma_{n}^{2}\left|a_{n}\right|^{2}<\infty
$$

where $f(z)=\sum_{n=0}^{+\infty} a_{n} z^{n} \in A_{\alpha}^{2}(\mathbb{D})$. Especially when $\alpha=0$, we write $A^{2}(\mathbb{D})=$ $A_{0}^{2}(\mathbb{D})$. In this case, $\gamma_{n}=\sqrt{\frac{1}{n+1}}$.

Received November 3, 2014; Revised February 19, 2015.

2010 Mathematics Subject Classification. Primary 47B35, 46E22.

Key words and phrases. reducing subspace, Toeplitz operator, polydisk.

This research is supported by NSFC (11271059,11201438), Research Fund for the Doctoral Program of Higher Education of China and Shandong Province Young Scientist Research Award Fund (BS2012SF031). 
Denote by $\mathbb{D}^{2}=\mathbb{D} \times \mathbb{D}$ the bidisk. The Bergman space $A^{2}\left(\mathbb{D}^{2}\right)$ is the space of all holomorphic functions in $L^{2}\left(\mathbb{D}^{2}, d \mu\right)$ where $d \mu(z)=d A\left(z_{1}\right) d A\left(z_{2}\right)$. For multi-index $\beta=\left(\beta_{1}, \beta_{2}\right)$, denote $z^{\beta}=z_{1}^{\beta_{1}} z_{2}^{\beta_{2}}$ and

$$
e_{\beta}=\frac{z^{\beta}}{\gamma_{\beta_{1}} \gamma_{\beta_{2}}} .
$$

Then $\left\{e_{\beta}\right\}_{\beta \succeq 0}\left(\beta \succeq 0\right.$ means that $\beta_{1} \geq 0$ and $\left.\beta_{2} \geq 0\right)$ is an orthogonal basis in $A^{2}\left(\mathbb{D}^{2}\right)$.

For a bounded measurable function $f \in L^{\infty}\left(\mathbb{D}^{2}\right)$, the Toeplitz operator with symbol $f$ is defined by $T_{f} h=P(f h)$ for every $h \in A^{2}\left(\mathbb{D}^{2}\right)$, where $P$ is the Bergman orthogonal projection from $L^{2}\left(\mathbb{D}^{2}, d \mu\right)$ onto $A^{2}\left(\mathbb{D}^{2}\right)$.

Recall that for a bounded linear operator $T$ on a Hilbert space $H$, a closed subspace $\mathcal{M}$ is called a reducing subspace of the operator $T$, if $T(\mathcal{M}) \subset \mathcal{M}$ and $T^{*}(\mathcal{M}) \subset \mathcal{M}$. A reducing subspace $\mathcal{M}$ is said to be minimal if there is no nonzero reducing subspace $\mathcal{N}$ such that $\mathcal{N}$ is properly contained in $\mathcal{M}$.

On the Bergman space over $\mathbb{D}$, it is proved that $T_{B}$ has just two non-trivial reducing subspaces $[13,16]$, where $B$ is the product of two Blaschke factors. In [12], M. Stessin and K. Zhu gave a complete description of the reducing subspaces of weighted unilateral shift operators of finite multiplicity. In particular, $T_{z^{n}}$ has $n$ distinct minimal reducing subspaces. If $B$ is a finite Blaschke product (order $n \geq 2$ ), the number of nontrivial minimal reducing subspaces of $T_{B}$ equals the number of connected components of the Riemann surface of $B^{-1} \circ B$ over $\mathbb{D}$ (see $[2,3,4,8,9,14]$ for details). Further, if $B$ is an infinite Blaschke product or a covering map, the relative research can be founded in $[5,6,7]$.

On the Bergman space of bidisk, Y. Lu and X. Zhou [10] characterized the reducing subspaces of $T_{z_{1}^{N} z_{2}^{N}}, T_{z_{1}^{N}}$ and $T_{z_{2}^{N}}$, respectively. The reducing subspaces of $T_{z_{1}^{N} z_{2}^{M}}$ on the weighted Bergman space $A_{\alpha}^{2}\left(\mathbb{D}^{2}\right)$ have been completely described in [11]. For $p=\alpha z^{k}+\beta w^{l}$, the minimal reducing subspaces of $T_{p}$ on $A^{2}\left(\mathbb{D}^{2}\right)$ and the commutant algebra $\mathcal{V}^{*}(p)=\left\{T_{p}, T_{p}^{*}\right\}^{\prime}$ was described in $[1,15]$.

In this paper, we mainly consider the reducing subspaces for the Toeplitz operator $T_{z_{1}^{N} \bar{z}_{2}^{M}}$ on the Bergman space $A^{2}\left(\mathbb{D}^{2}\right)$, where $N$ and $M$ are positive integers.

\section{Main results}

In this section, we will give a complete characterization of the reducing subspaces of $T_{z_{1}^{N} \bar{z}_{2}^{M}}$. To state our results, we need some notations and lemmas. Through out this paper, denote $T=T_{z_{1}^{N} \bar{z}_{2}^{M}}$, where $N$ and $M$ are positive integers. Denote by $[f]$ the reducing subspace of $T$ generated by $f \in A^{2}\left(\mathbb{D}^{2}\right)$. Let $\mathbb{N}$ be the set of all the nonnegative integers. 
By direct calculation, we know that

$$
\begin{gathered}
T^{h}\left(z_{1}^{k} z_{2}^{l}\right)=\left\{\begin{array}{cc}
\frac{\gamma_{l}^{2}}{\gamma_{l-h M}^{2}} z_{1}^{k+h N} z_{2}^{l-h M}, & \text { if } l \geq h M \\
0, & \text { if } l<h M
\end{array} ;\right. \\
T^{* h}\left(z_{1}^{k} z_{2}^{l}\right)=\left\{\begin{array}{cc}
\frac{\gamma_{k}^{2}}{\gamma_{k-h N}^{2}} z_{1}^{k-h N} z_{2}^{l+h M}, & \text { if } k \geq h N \\
0, & \text { if } k<h N
\end{array}\right.
\end{gathered}
$$

for $k, l, h \in \mathbb{N}$. Set

$$
\begin{aligned}
& E_{0}=\{(k, l) \in \mathbb{N} \times \mathbb{N}: 0 \leq k<N, 0 \leq l<M\}, \\
& E_{1}=\{(k, l) \in \mathbb{N} \times \mathbb{N}: k \geq 2 N\}, \\
& E_{2}=\{(k, l) \in \mathbb{N} \times \mathbb{N}: l \geq 2 M, 0 \leq k<2 N\}, \\
& E_{3}=\{(k, l) \in \mathbb{N} \times \mathbb{N}: N \leq k<2 N, M \leq l<2 M\}, \\
& E_{4}=\{(k, l) \in \mathbb{N} \times \mathbb{N}: 0 \leq k<N, M \leq l<2 M\}, \\
& E_{5}=\{(k, l) \in \mathbb{N} \times \mathbb{N}: 0 \leq l<M, N \leq k<2 N\} .
\end{aligned}
$$

Clearly,

$$
A^{2}\left(\mathbb{D}^{2}\right)=\bigoplus_{i=0}^{5} \overline{\operatorname{span}}\left\{z_{1}^{p} z_{2}^{q}:(p, q) \in E_{i}\right\} .
$$

Notice that $\mathcal{M}_{0}=\operatorname{span}\left\{z_{1}^{p} z_{2}^{q}:(p, q) \in E_{0}\right\}$ is a reducing subspace of $T$. To find other reducing subspaces, we first study the orthogonal decomposition of $z_{1}^{k} z_{2}^{l}$ with respect to $\mathcal{M}$.

Lemma 2.1. Suppose $\mathcal{M} \subset \mathcal{M}_{0}^{\perp}$ is a reducing subspace of $T$. Let $P_{\mathcal{M}}$ be the orthogonal projection from $A^{2}\left(\mathbb{D}^{2}\right)$ onto $\mathcal{M}$.

(i) If $(k, l) \in E_{1} \cup E_{2} \cup E_{3}$, then $P_{\mathcal{M}} z_{1}^{k} z_{2}^{l}=\lambda z_{1}^{k} z_{2}^{l}$ with some $\lambda \in \mathbb{C}$.

(ii) If $(k, l) \in E_{4}$, then

$$
P_{\mathcal{M}} z_{1}^{k} z_{2}^{l} \in \operatorname{span}\left\{z_{1}^{n} z_{2}^{m}:(n, m) \in E_{4}\right\} .
$$

(iii) If $(k, l) \in E_{5}$, then

$$
P_{\mathcal{M}} z_{1}^{k} z_{2}^{l} \in \operatorname{span}\left\{z_{1}^{n} z_{2}^{m}:(n, m) \in E_{5}\right\} .
$$

Proof. Let $k, l \in \mathbb{N}$. Since $\mathcal{M} \perp \mathcal{M}_{0},\left\langle P_{\mathcal{M}}\left(z_{1}^{k} z_{2}^{l}\right), z_{1}^{p} z_{2}^{q}\right\rangle=0$ for $(p, q) \in E_{0}$. In the following, we consider the inner product $\left\langle P_{\mathcal{M}}\left(z_{1}^{k} z_{2}^{l}\right), z_{1}^{p} z_{2}^{q}\right\rangle$ for $(p, q) \in$ $\bigcup_{i=1}^{5} E_{i}$

For every nonnegative integer $h$ satisfying $l \geq h M$,

$$
T^{h *} T^{h}\left(z_{1}^{k} z_{2}^{l}\right)=\frac{\gamma_{l}^{2} \gamma_{k+h N}^{2}}{\gamma_{l-h M}^{2} \gamma_{k}^{2}} z_{1}^{k} z_{2}^{l} .
$$

By computation,

$$
\frac{\gamma_{l}^{2} \gamma_{k+h N}^{2}}{\gamma_{l-h M}^{2} \gamma_{k}^{2}}\left\langle P_{\mathcal{M}}\left(z_{1}^{k} z_{2}^{l}\right), z_{1}^{p} z_{2}^{q}\right\rangle=\left\langle P_{\mathcal{M}} T^{h *} T^{h}\left(z_{1}^{k} z_{2}^{l}\right), z_{1}^{p} z_{2}^{q}\right\rangle
$$




$$
\begin{aligned}
& =\left\langle P_{\mathcal{M}}\left(z_{1}^{k} z_{2}^{l}\right), T^{h *} T^{h}\left(z_{1}^{p} z_{2}^{q}\right)\right\rangle \\
& =\left\{\begin{array}{cc}
\frac{\gamma_{q}^{2} \gamma_{p+h N}^{2}}{\gamma_{q-h M}^{2} \gamma_{p}^{2}}\left\langle P_{\mathcal{M}}\left(z_{1}^{k} z_{2}^{l}\right), z_{1}^{p} z_{2}^{q}\right\rangle, & q \geq h M \\
0, & q<h M .
\end{array}\right.
\end{aligned}
$$

Recall that $[s]=\max \{n \in \mathbb{Z}: n \leq s\}$ for real number $s$. By above equality, we get that if $\left\langle P_{\mathcal{M}}\left(z_{1}^{k} z_{2}^{l}\right), z_{1}^{p} z_{2}^{q}\right\rangle \neq 0$, then

$$
\frac{\gamma_{l}^{2} \gamma_{k+h N}^{2}}{\gamma_{l-h M}^{2} \gamma_{k}^{2}}=\frac{\gamma_{q}^{2} \gamma_{p+h N}^{2}}{\gamma_{q-h M}^{2} \gamma_{p}^{2}}
$$

for $0 \leq h \leq\left[\frac{l}{M}\right], q \geq\left[\frac{l}{M}\right] M$.

Equivalently,

$$
\frac{(k+1)(q+1)}{(p+1)(l+1)}=\frac{(k+1+h N)(q+1-h M)}{(p+1+h N)(l+1-h M)}
$$

for $0 \leq h \leq\left[\frac{l}{M}\right], q \geq\left[\frac{l}{M}\right] M$.

(i) If $(k, l) \in E_{1} \cup E_{2} \cup E_{3}$, we will show that the equality (2) holds if and only if $p=k$ and $q=l$.

Case one: $l \geq 2 M$. Let $g_{1}(\lambda)=(k+1)(q+1)(p+1+\lambda N)(l+1-\lambda M)$, $g_{2}(\lambda)=(p+1)(l+1)(k+1+\lambda N)(q+1-\lambda M)$ and $g(\lambda)=g_{1}(\lambda)-g_{2}(\lambda)$. Since $l \geq 2 M$, we have $g(0)=g(1)=g(2)=0$. Considering $g(\lambda)$ is a quadratic polynomial, we have $g(\lambda) \equiv 0$ on $\mathbb{C}$. Therefore, $g_{1}$ and $g_{2}$ have the same zeros, i.e.,

$$
\left\{\begin{aligned}
(k+1)(q+1) N M & =(p+1)(l+1) N M \\
(k+1)(q+1) \frac{p+1}{N} & =(p+1)(l+1) \frac{k+1}{N} \\
(k+1)(q+1) \frac{l+1}{M} & =(p+1)(l+1) \frac{q+1}{M} .
\end{aligned}\right.
$$

It follows that $p=k$ and $q=l$.

Case two: $k \geq 2 N$. Replacing $T^{*} T$ by $T T^{*}$ in Case one, we can get the desire result. The details are listed as follows.

Since

we know that

$$
T^{h} T^{h *}\left(z_{1}^{k} z_{2}^{l}\right)=\frac{\gamma_{k}^{2} \gamma_{l+h M}^{2}}{\gamma_{k-h N}^{2} \gamma_{l}^{2}} z_{1}^{k} z_{2}^{l}, \forall 0 \leq h \leq\left[\frac{k}{N}\right],
$$

$$
\begin{aligned}
\frac{\gamma_{k}^{2} \gamma_{l+h M}^{2}}{\gamma_{k-h N}^{2} \gamma_{l}^{2}}\left\langle P_{\mathcal{M}}\left(z_{1}^{k} z_{2}^{l}\right), z_{1}^{p} z_{2}^{q}\right\rangle & =\left\langle P_{\mathcal{M}} T^{h} T^{h *}\left(z_{1}^{k} z_{2}^{l}\right), z_{1}^{p} z_{2}^{q}\right\rangle \\
& =\left\langle P_{\mathcal{M}}\left(z_{1}^{k} z_{2}^{l}\right), T^{h} T^{h *}\left(z_{1}^{p} z_{2}^{q}\right)\right\rangle \\
& =\left\{\begin{array}{ccc}
\frac{\gamma_{p}^{2} \gamma_{q+h M}^{2}}{\gamma_{p-h N}^{2} \gamma_{q}^{2}}\left\langle P_{\mathcal{M}}\left(z_{1}^{k} z_{2}^{l}\right), z_{1}^{p} z_{2}^{q}\right\rangle & \text { if } & p \geq h N \\
0 & \text { if } & p<h N .
\end{array}\right.
\end{aligned}
$$

Therefore, $\left\langle P_{\mathcal{M}}\left(z_{1}^{k} z_{2}^{l}\right), z_{1}^{p} z_{2}^{q}\right\rangle \neq 0$ will give that

$$
\frac{\gamma_{k}^{2} \gamma_{l+h M}^{2}}{\gamma_{k-h N}^{2} \gamma_{l}^{2}}=\frac{\gamma_{p}^{2} \gamma_{q+h M}^{2}}{\gamma_{p-h N}^{2} \gamma_{q}^{2}}
$$


for $0 \leq h \leq\left[\frac{k}{N}\right]$ and $p \geq\left[\frac{k}{N}\right] N$. Equivalently,

$$
\frac{(k+1)(q+1)}{(p+1)(l+1)}=\frac{(k+1-h N)(q+1+h M)}{(p+1-h N)(l+1+h M)}
$$

for $0 \leq h \leq\left[\frac{k}{N}\right]$ and $p \geq\left[\frac{k}{N}\right] N$. So when $k \geq 2 N$, the above equality follows for $h=0,1,2$. In this case we will get $p=k$ and $q=l$ by the same arguments as the case $l \geq 2 M$ has done.

Case three: $(k, l) \in E_{3}=\left\{(n, m) \in \mathbb{N}^{2}: N \leq n<2 N, M \leq m<2 M\right\}$. In this case, $\left[\frac{k}{N}\right] \geq 1$ and $\left[\frac{l}{M}\right] \geq 1$. Then equalities (3) and (5) hold for $h=0,1$. Recall that $g(\lambda)=g_{1}(\lambda)-g_{2}(\lambda)$, where $g_{1}(\lambda)=(k+1)(q+1)(p+$ $1+\lambda N)(l+1-\lambda M)$ and $g_{2}(\lambda)=(p+1)(l+1)(k+1+\lambda N)(q+1-\lambda M)$. We get $g(0)=g(1)=g(-1)=0$. Therefore, we obtain that $p=k$ and $q=l$.

(ii) Suppose that $(k, l) \in E_{4}$. We need only prove that

$$
P_{\mathcal{M}}\left(z_{1}^{k} z_{2}^{l}\right) \perp \overline{\operatorname{span}}\left\{z_{1}^{n} z_{2}^{m}:(n, m) \in\left(\bigcup_{i=1}^{3} E_{i}\right) \bigcup E_{5}\right\} .
$$

If $(n, m) \in E_{1} \cup E_{2} \cup E_{3}$, the conclusion (i) implies that $P_{\mathcal{M}} z_{1}^{n} z_{2}^{m}=\lambda z_{1}^{n} z_{2}^{m}$ for some $\lambda \in \mathbb{C}$. Thus

$$
\left\langle P_{\mathcal{M}} z_{1}^{k} z_{2}^{l}, z_{1}^{n} z_{2}^{m}\right\rangle=\left\langle z_{1}^{k} z_{2}^{l}, P_{\mathcal{M}} z_{1}^{n} z_{2}^{m}\right\rangle=\bar{\lambda}\left\langle z_{1}^{k} z_{2}^{l}, z_{1}^{n} z_{2}^{m}\right\rangle=0 .
$$

That is, $P_{\mathcal{M}} z_{1}^{k} z_{2}^{l} \perp \overline{\operatorname{span}}\left\{z_{1}^{p} z_{2}^{q}:(p, q) \in E_{1} \cup E_{2} \cup E_{3}\right\}$.

If $(n, m) \in E_{5}=\{(k, l) \in \mathbb{N} \times \mathbb{N}: 0 \leq l<M, N \leq k<2 N\}$,

$$
\begin{aligned}
\left\langle P_{\mathcal{M}} z_{1}^{k} z_{2}^{l}, z_{1}^{n} z_{2}^{m}\right\rangle & =\frac{\gamma_{l-M}^{2} \gamma_{k}^{2}}{\gamma_{l}^{2} \gamma_{k+N}^{2}}\left\langle P_{\mathcal{M}} T^{*} T z_{1}^{k} z_{2}^{l}, z_{1}^{n} z_{2}^{m}\right\rangle \\
& =\frac{\gamma_{l-M}^{2} \gamma_{k}^{2}}{\gamma_{l}^{2} \gamma_{k+N}^{2}}\left\langle T P_{\mathcal{M}} z_{1}^{k} z_{2}^{l}, T z_{1}^{n} z_{2}^{m}\right\rangle=0,
\end{aligned}
$$

where the last equality comes from $\operatorname{span}\left\{z_{1}^{p} z_{2}^{q}:(p, q) \in E_{5}\right\} \subseteq \operatorname{Ker} T$. Thus $P_{\mathcal{M}} z_{1}^{k} z_{2}^{l} \perp \operatorname{span}\left\{z_{1}^{p} z_{2}^{q}:(p, q) \in E_{5}\right\}$.

(iii) Replacing $T^{*} T$ by $T T^{*}$ in (ii), we get the desired result.

Remark 2.1. Let $\mathcal{M} \subset \mathcal{M}_{0}^{\perp}$ is a nonzero reducing subspace of $T$. In (i) of Lemma 2.1, we indeed get that $\lambda=0$ or 1 , that is $z_{1}^{k} z_{2}^{l} \in \mathcal{M}$ or $z_{1}^{k} z_{2}^{l} \in \mathcal{M}^{\perp}$ for each $(k, l) \in E_{1} \cup E_{2} \cup E_{3}$.

If $z_{1}^{k} z_{2}^{l} \in \mathcal{M}$, then

$$
\left[z_{1}^{k} z_{2}^{l}\right]=\operatorname{span}\left\{z_{1}^{k-h N} z_{2}^{l+h M}: k-h N \geq 0, l+h M \geq 0, h \in \mathbb{Z}\right\}
$$

is a minimal reducing subspace of $T$, containing in $\mathcal{M}$. Moreover, if $z_{1}^{k} z_{2}^{l}, z_{1}^{p} z_{2}^{q} \in$ $\mathcal{M}$ and $(k, l),(p, q) \in E_{1} \cup E_{2} \cup E_{3}$, then it's clear that either $\left[z_{1}^{k} z_{2}^{l}\right] \perp\left[z_{1}^{p} z_{2}^{q}\right]$ or $\left[z_{1}^{k} z_{2}^{l}\right]=\left[z_{1}^{p} z_{2}^{q}\right]$. So for any non-zero function $f(z)=\sum_{(k, l) \in E_{1} \cup E_{2} \cup E_{3}} a_{k, l} z_{1}^{k} z_{2}^{l}$, $[f]$ is the direct sum of some minimal reducing subspace as (6).

We define two equivalences on $E_{4}$ and $E_{5}$ respectively by: 
(i) for $(p, q),(k, l) \in E_{4},(p, q) \sim_{1}(k, l) \Leftrightarrow \frac{(k+1)(q+1)}{(p+1)(l+1)}=\frac{(k+1+N)(q+1-M)}{(p+1+N)(l+1-M)}$;

(ii) for $(p, q),(k, l) \in E_{5},(p, q) \sim_{2}(k, l) \Leftrightarrow \frac{(k+1)(q+1)}{(p+1)(l+1)}=\frac{(k+1-N)(q+1+M)}{(p+1-N)(l+1+M)}$.

It is easy to check that

(i) $(p, q) \in E_{4} \Leftrightarrow(p+N, q-M) \in E_{5}$;

(ii) for $(p, q),(k, l) \in E_{4},(p, q) \sim_{1}(k, l) \Leftrightarrow(p+N, q-M) \sim_{2}(k+N, l-M)$;

(iii) for $(p, q),(k, l) \in E_{5},(p, q) \sim_{2}(k, l) \Leftrightarrow(p-N, q+M) \sim_{1}(k-N, l+M)$.

For $(n, m) \in E_{4}$ and $(k, l) \in E_{5}$, let

$$
\begin{array}{r}
P_{n, m}: A^{2}\left(\mathbb{D}^{2}\right) \rightarrow \operatorname{span}\left\{z_{1}^{p} z_{2}^{q}:(p, q) \sim_{1}(n, m),(p, q) \in E_{4}\right\}, \\
\left.Q_{k, l}: A^{2}\left(\mathbb{D}^{2}\right) \rightarrow \operatorname{span}\left\{z_{1}^{k} z_{2}^{l}:(p, q) \sim_{2}(k, l),(p, q)\right) \in E_{5}\right\}
\end{array}
$$

be two orthogonal projections. For $f \in A^{2}\left(\mathbb{D}^{2}\right)$ and $P_{n, m} f \neq 0$, we have

$$
\left[P_{n, m} f\right]=\operatorname{span}\left\{P_{n, m} f, T P_{n, m} f\right\},
$$

since $T^{*} P_{n, m} f=0, T^{2} P_{n, m} f=0$ and $T^{*} T P_{n, m} f=\frac{\gamma_{m}^{2} \gamma_{n+N}^{2}}{\gamma_{m-M}^{2} \gamma_{n}^{2}} P_{n, m} f$. Similarly, if $f \in \mathcal{M}$ and $Q_{k, l} f \neq 0$, then

$$
\left[Q_{k, l} f\right]=\operatorname{span}\left\{Q_{k, l} f, T^{*} Q_{k, l} f\right\} .
$$

Lemma 2.2. Let $\mathcal{M} \subset \mathcal{M}_{0}^{\perp}$ be a reducing subspace of $T$ and $(n, m) \in E_{4}$. Then the following statements hold.

(a) If $f \in \mathcal{M}$, then $\left[P_{n, m} f\right] \subset \mathcal{M}$ and $\left[Q_{n+N, m-M} f\right] \subset \mathcal{M}$.

(b) If $f_{1}, f_{2} \in P_{n, m} \mathcal{M}$ and $f_{1} \perp f_{2}$, then $\left[f_{1}\right] \perp\left[f_{2}\right]$.

(c) $P_{n, m} T^{*} f=T^{*} Q_{n+N, m-M} f$ and $T P_{n, m} f=Q_{n+N, m-M} T f, \forall f \in \mathcal{M}$.

(d) If $f \in \mathcal{M}$, then $\left[P_{n, m} f\right]=\left[Q_{n+N, m-M} T f\right]$ and $\left[Q_{n+N, m-M} f\right]=$ $\left[P_{n, m} T^{*} f\right]$.

(e) $P_{n, m} \mathcal{M} \oplus Q_{n+N, m-M} \mathcal{M} \subset \mathcal{M}$ is a reducing subspace of $T$.

Proof. (a) For every $f \in \mathcal{M}$, we know that $P_{\mathcal{M}} P_{n, m} f=P_{n, m} f$, since $P_{\mathcal{M}} P_{n, m}$ $=P_{n, m} P_{\mathcal{M}}$, which obtained by the following simple facts:

(i) if $(k, l) \in E_{4}$, then $P_{\mathcal{M}} z_{1}^{k} z_{2}^{l} \in \operatorname{span}\left\{z_{1}^{p} z_{2}^{q}:(p, q) \in E_{4}\right\}$;

(ii) if $(k, l) \notin E_{4}$, then $P_{\mathcal{M}} z_{1}^{k} z_{2}^{l} \perp \operatorname{span}\left\{z_{1}^{p} z_{2}^{q}:(p, q) \in E_{4}\right\}$.

So $P_{n, m} f \in \mathcal{M}$, which implies that $\left[P_{n, m} f\right] \subset \mathcal{M}$.

Similarly, we have $P_{\mathcal{M}} Q_{n+N, m-M} f=Q_{n+N, m-M} f$, which shows that $Q_{n+N, m-M} f \in \mathcal{M}$. Thus $\left[Q_{n+N, m-M} f\right] \subset \mathcal{M}$.

(b) It is clear that $T f_{1}, T f_{2} \in \operatorname{span}\left\{z_{1}^{k} z_{2}^{l}:(k, l) \in E_{5}\right\}$ and

$$
\left\langle T f_{1}, T f_{2}\right\rangle=\left\langle T^{*} T f_{1}, f_{2}\right\rangle=\frac{\gamma_{n+N}^{2} \gamma_{m}^{2}}{\gamma_{n}^{2} \gamma_{m-M}^{2}}\left\langle f_{1}, f_{2}\right\rangle=0 .
$$

Equality (7) shows that

$$
\left[f_{1}\right]=\operatorname{span}\left\{f_{1}, T f_{1}\right\},\left[f_{2}\right]=\operatorname{span}\left\{f_{2}, T f_{2}\right\} .
$$

So $\left[f_{1}\right] \perp\left[f_{2}\right]$. 
(c) For every $(n, m) \in E_{4}$, let

$$
\begin{aligned}
\mathcal{M}_{n, m} & =\operatorname{span}\left\{z_{1}^{k} z_{2}^{l}:(k, l) \sim_{1}(n, m),(k, l) \in E_{4}\right\}, \\
\mathcal{M}_{n+N, m-M} & =\operatorname{span}\left\{z_{1}^{k} z_{2}^{l}:(k, l) \sim_{2}(n+N, m-M),(k, l) \in E_{5}\right\} .
\end{aligned}
$$

Then $\mathcal{M}_{n, m}$ and $\mathcal{M}_{n+N, m-M}$ are finite dimension, and the following statements hold:

(i) $T \mathcal{M}_{n, m}=\mathcal{M}_{n+N, m-M}$ and $T^{*} \mathcal{M}_{n+N, m-M}=\mathcal{M}_{n, m}$;

(ii) $T\left(\mathcal{M}_{n, m}^{\perp}\right) \subset \mathcal{M}_{n+N, m-M}^{\perp}$ and $T^{*}\left(\mathcal{M}_{n+N, m-M}^{\perp}\right) \subset \mathcal{M}_{n, m}^{\perp}$.

Therefore, $T P_{n, m} f=Q_{n+N, m-M} T f$ and $P_{n, m} T^{*} f=T^{*} Q_{n+N, m-M} f$ for any $f \in \mathcal{M}$.

(d) By equality (7), conclusion (c) and

$$
T^{*} T P_{n, m} f=\frac{\gamma_{n+N}^{2} \gamma_{m}^{2}}{\gamma_{n}^{2} \gamma_{m-M}^{2}} P_{n, m} f,
$$

we have

$$
\begin{aligned}
{\left[Q_{n+N, m-M} T f\right] } & =\operatorname{span}\left\{Q_{n+N, m-M} T f, T^{*} Q_{n+N, m-M} T f\right\} \\
& =\operatorname{span}\left\{T P_{n, m} f\right\} \oplus \operatorname{span}\left\{T^{*} T P_{n, m} f\right\} \\
& =\operatorname{span}\left\{T P_{n, m} f\right\} \oplus \operatorname{span}\left\{P_{n, m} f\right\} \\
& =\left[P_{n, m} f\right] .
\end{aligned}
$$

Similarly, $\left[Q_{n+N, m-M} f\right]=\left[P_{n, m} T^{*} f\right]$ comes from equality (8), conclusion (c) and

$$
T T^{*} Q_{n+n, m-M} f=\frac{\gamma_{n+N}^{2} \gamma_{m}^{2}}{\gamma_{n}^{2} \gamma_{m-M}^{2}} Q_{n+N, m-M} f .
$$

(e) By equalities (9), (10) and conclusion (c), we have

$$
\begin{aligned}
Q_{n+N, m-M} \mathcal{M} & =T T^{*}\left(Q_{n+N, m-M} \mathcal{M}\right)=T P_{n, m} T^{*} \mathcal{M} \\
P_{n, m} \mathcal{M} & =T^{*} T\left(P_{n, m} \mathcal{M}\right)=T^{*} Q_{n+N, m-M} T \mathcal{M}
\end{aligned}
$$

Therefore, we only need to show that $P_{n, m} \mathcal{M} \oplus Q_{n+N, m-M} \mathcal{M}$ is an invariant subspace of $T$ and $T^{*}$. In fact,

$$
T\left(P_{n, m} \mathcal{M} \oplus Q_{n+N, m-M} \mathcal{M}\right)=T P_{n, m} \mathcal{M}=Q_{n+N, m-M} \mathcal{M},
$$

where the last equality comes from $T P_{n, m} f=Q_{n+N, m-M} T f \in Q_{n+N, m-M} \mathcal{M}$ and $Q_{n+N, m-M} f \in T P_{n, m} T^{*} \mathcal{M} \subset T P_{n, m} \mathcal{M}$ for all $f \in \mathcal{M}$. Therefore,

$$
T\left(P_{n, m} \mathcal{M} \oplus Q_{n+N, m-M} \mathcal{M}\right) \subset P_{n, m} \mathcal{M} \oplus Q_{n+N, m-M} \mathcal{M}
$$

Similarly, we can prove that

$$
T^{*}\left(P_{n, m} \mathcal{M} \oplus Q_{n+N, m-M} \mathcal{M}\right)=T^{*} Q_{n+N, m-M} \mathcal{M}=P_{n, m} \mathcal{M} .
$$

So we finish the proof. 
Remark 2.2. In the prove of (e), we also get that

$$
\left[P_{n, m} \mathcal{M}\right]=P_{n, m} \mathcal{M} \oplus Q_{n+N, m-M} \mathcal{M}=\left[Q_{n+N, m-M} \mathcal{M}\right],
$$

where $\left[P_{n, m} \mathcal{M}\right]$ and $\left[Q_{n+N, m-M} \mathcal{M}\right]$ are the reducing subspaces generated by $P_{n, m} \mathcal{M}$ and $Q_{n+N, m-M} \mathcal{M}$, respectively.

Theorem 2.1. Let $\mathcal{M} \subset \mathcal{M}_{0}^{\perp}$ be a non-zero reducing subspace of $T$ on the bidisk. Then $\mathcal{M}=\mathcal{M}_{1} \oplus \mathcal{M}_{2}$, where

(i) $\mathcal{M}_{1}$ is a direct sum of minimal reducing subspace $\left[z_{1}^{p} z_{2}^{q}\right]$ with $z_{1}^{p} z_{2}^{q} \in \mathcal{M}$ for some $(p, q) \in E_{1} \cup E_{2} \cup E_{3}$;

(ii) $\mathcal{M}_{2}$ is a direct sum of minimal reducing subspace $[f]$ with $f \in P_{n, m} \mathcal{M}$ for some $(n, m) \in E_{4}$.

Proof. Firstly, we prove that

$$
\mathcal{M}=\mathcal{M}_{1} \bigoplus \bigoplus_{(n, m) \in E}\left(P_{n, m} \mathcal{M} \bigoplus Q_{n+N, m-M} \mathcal{M}\right),
$$

where $\mathcal{M}_{1}=\bigoplus_{(p, q) \in \Lambda}\left[z_{1}^{p} z_{2}^{q}\right]$ with $\Lambda=\left\{(p, q) \in E_{1} \cup E_{2} \cup E_{3}: z_{1}^{p} z_{2}^{q} \in \mathcal{M}\right\}$, and $E$ is the partition of $E_{4}$ by the equivalence $\sim_{1}$. Set $\mathcal{H}_{n, m}=P_{n, m} \mathcal{M} \bigoplus Q_{n+N, m-M} \mathcal{M}$.

On the one hand, $\mathcal{M}_{1} \bigoplus \bigoplus_{(n, m) \in E} \mathcal{H}_{n, m} \subset \mathcal{M}$, since $\mathcal{M}_{1} \subset \mathcal{M}$ is a reducing subspace of $T$, and conclusion (e) in Lemma 2.2 implies that $\bigoplus_{(n, m) \in E} \mathcal{H}_{n, m} \subset$ $\mathcal{M}$. On the other hand, for $g=g_{1}+g_{2} \in \mathcal{M}$ with

(13) $g_{1}(z)=\sum_{(p, q) \in E_{1} \cup E_{2} \cup E_{3}} a_{p, q} z_{1}^{p} z_{2}^{q}, g_{2}(z)=\sum_{(p, q) \in E_{4} \cup E_{5}} a_{p, q} z_{1}^{p} z_{2}^{q}$.

Remark 2.1 shows that $g_{1} \in \mathcal{M}_{1} \subset \mathcal{M}$, which implies that $g_{2}=g-g_{1} \in \mathcal{M}$. Therefore, $g_{2}=\sum_{(n, m) \in E}\left(P_{n, m} g_{2}+Q_{n+N, m-M} g_{2}\right) \in \bigoplus_{(n, m) \in E} \mathcal{H}_{n, m}$. It follows that $\mathcal{M}$ is in the direct sum of $\mathcal{M}_{1}$ and $\left\{\mathcal{H}_{n, m}\right\}$ with $(n, m) \in E$. So we have equality (12) holds.

Secondly, for each $(n, m) \in E_{4}$, we prove that $\mathcal{H}_{n, m}$ is the direct sum of minimal reducing subspaces as $[f]=\operatorname{span}\{f, T f\}$ with $f \in P_{n, m} \mathcal{M}$. There are some steps in the proof.

Step 1. Take $0 \neq f_{1} \in P_{n, m} \mathcal{M}$. Then $\left[f_{1}\right]=\operatorname{span}\left\{f_{1}, T f_{1}\right\} \subset \mathcal{H}_{n, m}$.

Step 2. If $P_{n, m} \mathcal{M} \neq \mathbb{C} f_{1}$, take $0 \neq f_{2} \in P_{n, m} \mathcal{M} \ominus \mathbb{C} f_{1}$. Then

$$
\left[f_{2}\right]=\operatorname{span}\left\{f_{2}, T f_{2}\right\} \subset \mathcal{H}_{n, m} \ominus\left[f_{1}\right] .
$$

Step 3. If $P_{n, m} \mathcal{M} \neq \operatorname{span}\left\{f_{1}, f_{2}\right\}$, take $0 \neq f_{3} \in P_{n, m} \mathcal{M} \ominus \operatorname{span}\left\{f_{1}, f_{2}\right\}$. Then

$$
\left[f_{3}\right]=\operatorname{span}\left\{f_{3}, T f_{3}\right\} \subset \mathcal{H}_{n, m} \ominus\left[f_{1}\right] \ominus\left[f_{2}\right] .
$$

If $P_{n, m} \mathcal{M} \neq \operatorname{span}\left\{f_{1}, f_{2}, f_{3}\right\}$, continue this process. This process will stop in finite steps, since the dimension of $\mathcal{H}_{n, m}$ is finite. Thus, we finish the proof. 
Remark 2.3. In particular, if $\mathcal{M}$ is a reducing subspace generated by $g=$ $g_{1}+g_{2} \in A^{2}\left(\mathbb{D}^{2}\right)$ as in $(13)$, then $[g]=\left[g_{1}\right] \oplus\left[g_{2}\right]$ and

$$
\left[g_{2}\right]=\bigoplus_{(n, m) \in E}\left[P_{n, m} g, Q_{n+N, m-M} g\right],
$$

where $\left[P_{n, m} g, Q_{n+N, m-M} g\right]$ is the reducing subspace generated by $P_{n, m} g$ and $Q_{n+N, m-M}$. By conclusions (a) and (d) in Lemma 2.2 and equalities in (11), we get $\left[P_{n, m} g, Q_{n+N, m-M} g\right]=\left[P_{n, m} g, P_{n, m} T^{*} g\right]=\operatorname{span}\left\{P_{n, m} g, P_{n, m} T^{*} g\right\} \oplus$ $\operatorname{span}\left\{Q_{n+N, m-M} g, Q_{n+N, m-M} T g\right\}$.

Notice that $\operatorname{span}\left\{P_{n, m} g, P_{n, m} T^{*} g\right\}$ has an orthonormal basis $\left\{e_{1}, \ldots, e_{k}\right\}$, since the dimension of $\operatorname{span}\left\{P_{n, m} g, P_{n, m} T^{*} g\right\}$ is finite. Conclusion (b) in Lemma 2.2 shows that $\left[e_{i}\right] \perp\left[e_{j}\right]$ for $i \neq j$. Then we get

$$
\left[P_{n, m} g, P_{n, m} T^{*} g\right]=\bigoplus_{j=1}^{k}\left[e_{j}\right]=\bigoplus_{j=1}^{k} \operatorname{span}\left\{e_{j}, T e_{j}\right\} .
$$

Similarly, we can prove that

$$
\left[g_{2}\right]=\bigoplus_{(n, m) \in E}\left[Q_{n+N, m-M} g, Q_{n+N, m-M} T g\right]
$$

and

$$
\left[Q_{n+N, m-M} g, Q_{n+N, m-M} T g\right]=\bigoplus_{j=1}^{l}\left[h_{j}\right]=\bigoplus_{j=1}^{l} \operatorname{span}\left\{h_{j}, T^{*} h_{j}\right\},
$$

where $\left\{h_{1}, \ldots, h_{l}\right\}$ is an orthonormal basis of

$$
\operatorname{span}\left\{Q_{n+N, m-M} g, Q_{n+N, m-M} T g\right\} .
$$

In the last part of this paper, we give some examples of the reducing subspaces of $T_{z_{1}^{N} \bar{z}_{2}^{M}}$ for the case that $N=M$ and $N \neq M$, respectively.

Example 2.1. Fix $a, b, c, d, e \in \mathbb{C}$ with $e \neq 0$. Let

$$
f\left(z_{1}, z_{2}\right)=a z_{1}^{9} z_{2}^{14}+b z_{1}^{7} z_{2}^{15}+c z_{1}^{5} z_{2}^{17}+d z_{1}^{4} z_{2}^{19}+e z_{1}^{11} z_{2}^{12}
$$

and $[f]$ be the reducing subspace of $T_{z_{1}^{10} \bar{z}_{2}^{10}}$ generated by $f$. Then

$$
[f]=\operatorname{span}\left\{f_{1}, f_{2}\right\} \oplus \operatorname{span}\left\{z_{1}^{11+10 h} z_{2}^{12-10 h}: h=-1,0,1\right\}
$$

where

$$
\begin{aligned}
& f_{1}\left(z_{1}, z_{2}\right)=a z_{1}^{9} z_{2}^{14}+b z_{1}^{7} z_{2}^{15}+c z_{1}^{5} z_{2}^{17}+d z_{1}^{4} z_{2}^{19} \\
& f_{2}\left(z_{1}, z_{2}\right)=\frac{a}{3} z_{1}^{19} z_{2}^{4}+\frac{3 b}{8} z_{1}^{17} z_{2}^{5}+\frac{4 c}{9} z_{1}^{15} z_{2}^{7}+\frac{d}{2} z_{1}^{14} z_{2}^{9} .
\end{aligned}
$$

Proof. Notice that $(11,12) \in E_{3}$ and $(9,14) \in E_{4}$. A direct computation shows that $(9,14) \sim_{1}(7,15) \sim_{1}(5,17) \sim_{1}(4,19)$. Remark 2.1 implies that $f_{1}=$ $P_{4,19} f$ and $z_{1}^{11} z_{2}^{12}$ are in $\mathcal{M}$. As in Remark 2.3 , there is $\operatorname{span}\left\{P_{4,19} f, P_{4,19} T^{*} f\right\}$ $=\left[f_{1}\right]=\operatorname{span}\left\{f_{1}, f_{2}\right\}$. Therefore we get the desired result. 
Example 2.2. Let $f\left(z_{1}, z_{2}\right)=z_{1}^{4} z_{2}^{14}+z_{1}^{7} z_{2}^{7}+z_{1}^{3} z_{2}^{15}$ and $[f]$ be the reducing subspace of $T_{z_{1}^{5} \bar{z}_{2}^{10}}$ generated by $f$. Then

$$
[f]=\operatorname{span}\left\{z_{1}^{4} z_{2}^{14}+z_{1}^{3} z_{2}^{15}, \frac{1}{3} z_{1}^{9} z_{2}^{4}+\frac{3}{8} z_{1}^{8} z_{2}^{5}\right\} \oplus \operatorname{span}\left\{z_{1}^{7} z_{2}^{7}, z_{1}^{2} z_{2}^{17}\right\} .
$$

Proof. Notice that $(7,7) \in E_{5},(4,14),(3,15) \in E_{4}$ and $(4,14) \sim_{1}(3,15)$. Let $f_{1}=P_{4,14} f=z_{1}^{4} z_{2}^{14}+z_{1}^{3} z_{2}^{15}$ and $f_{2}=Q_{7,7} f=z_{1}^{7} z_{2}^{7}$. Then $\left[P_{4,14} f, P_{4,14} T^{*} f\right]=$ $\left[f_{1}\right]=\operatorname{span}\left\{z_{1}^{4} z_{2}^{14}+z_{1}^{3} z_{2}^{15}, \frac{1}{3} z_{1}^{9} z_{2}^{4}+\frac{3}{8} z_{1}^{8} z_{2}^{5}\right\},\left[P_{2,17} f, P_{2,17} T^{*} f\right]=\left[Q_{7,7} f, Q_{7,7} T f\right]$ $=\left[f_{2}\right]=\operatorname{span}\left\{z_{1}^{7} z_{2}^{7}, z_{1}^{2} z_{2}^{17}\right\}$. Then we finish the proof.

Example 2.3. Let $f\left(z_{1}, z_{2}\right)=z_{1}^{3} z_{2}^{8}+z_{1}^{7} z_{2}^{3}$, and $[f]$ be the reducing subspace of $T_{z_{1}^{4} \bar{z}_{2}^{5}}$ generated by $f$. Then

$$
[f]=\operatorname{span}\left\{z_{1}^{3} z_{2}^{8}, z_{1}^{7} z_{2}^{3}\right\} .
$$

Proof. Notice that $(3,8) \in E_{4},(7,3) \in E_{5}$. It is easy to check that $T_{z_{1}^{4} \bar{z}_{2}^{5}} z_{1}^{3} z_{2}^{8}=$ $\frac{4}{9} z_{1}^{7} z_{2}^{3}$ and $T_{z_{1}^{4} \bar{z}_{2}^{5}}^{*} z_{1}^{7} z_{2}^{3}=\frac{1}{2} z_{1}^{3} z_{2}^{8}$. So $\left[z_{1}^{3} z_{2}^{8}\right]=\left[z_{1}^{7} z_{2}^{3}\right]=\operatorname{span}\left\{z_{1}^{3} z_{2}^{8}, z_{1}^{7} z_{2}^{3}\right\}$. It means that $[f]=\operatorname{span}\left\{z_{1}^{3} z_{2}^{8}, z_{1}^{7} z_{2}^{3}\right\}$.

Example 2.4. Let $f\left(z_{1}, z_{2}\right)=z_{1}^{2} z_{2}^{17}+z_{1}^{4} z_{2}^{14}+z_{1}^{9} z_{2}^{4}+z_{1}^{3} z_{2}^{15}+z_{1}^{8} z_{2}^{5}$ and $[f]$ be the reducing subspace of $T_{z_{1}^{5}} \bar{z}_{2}^{10}$ generated by $f$. Then

$$
\begin{aligned}
{[f] } & =\left[z_{1}^{2} z_{2}^{17}\right] \oplus\left[z_{1}^{4} z_{2}^{14}\right] \oplus\left[z_{1}^{3} z_{2}^{15}\right] \\
& =\left[z_{1}^{2} z_{2}^{17}\right] \oplus\left[z_{1}^{4} z_{2}^{14}+z_{1}^{3} z_{2}^{15}\right] \oplus\left[z_{1}^{4} z_{2}^{14}-\frac{64}{75} z_{1}^{3} z_{2}^{15}\right] \\
& =\left[z_{1}^{7} z_{2}^{7}\right] \oplus\left[z_{1}^{9} z_{2}^{4}+z_{1}^{8} z_{2}^{5}\right] \oplus\left[z_{1}^{9} z_{2}^{4}-\frac{27}{25} z_{1}^{8} z_{2}^{5}\right] .
\end{aligned}
$$

Proof. Notice that $(2,17),(4,14),(3,15) \in E_{4},(9,4),(8,5) \in E_{5}$ and

$$
(4,14) \sim_{1}(3,15),(9,4) \sim_{2}(8,5) .
$$

(i) Since $P_{4,14} T^{*} f=T^{*}\left(z_{1}^{9} z_{2}^{4}+z_{1}^{8} z_{2}^{5}\right)=\frac{1}{2} z_{1}^{4} z_{2}^{14}+\frac{4}{9} z_{1}^{3} z_{2}^{15}$, we have $\operatorname{span}\left\{P_{4,14} f, P_{4,14} T^{*} f\right\}=\operatorname{span}\left\{z_{1}^{4} z_{2}^{14}, z_{1}^{3} z_{2}^{15}\right\}$.

Therefore,

$$
\begin{aligned}
{[f] } & =\left[z_{1}^{2} z_{2}^{17}\right] \oplus\left[z_{1}^{4} z_{2}^{14}\right] \oplus\left[z_{1}^{3} z_{2}^{15}\right] \\
& =\operatorname{span}\left\{z_{1}^{2} z_{2}^{17}, z_{1}^{7} z_{2}^{7}\right\} \oplus \operatorname{span}\left\{z_{1}^{4} z_{2}^{14}, z_{1}^{9} z_{2}^{4}\right\} \oplus \operatorname{span}\left\{z_{1}^{3} z_{2}^{15}, z_{1}^{8} z_{2}^{5}\right\} .
\end{aligned}
$$

(ii) It is easy to check that $\left\langle z_{1}^{4} z_{2}^{14}-\frac{64}{75} z_{1}^{3} z_{2}^{15}, z_{1}^{4} z_{2}^{14}+z_{1}^{3} z_{2}^{15}\right\rangle=0$ and $\operatorname{span}\left\{P_{4,14} f, P_{4,14} T^{*} f\right\}=\operatorname{span}\left\{z_{1}^{4} z_{2}^{14}+z_{1}^{3} z_{2}^{15}, z_{1}^{4} z_{2}^{14}-\frac{64}{75} z_{1}^{3} z_{2}^{15}\right\}$.

So $[f]=\left[z_{1}^{4} z_{2}^{14}+z_{1}^{3} z_{2}^{15}\right] \oplus\left[z_{1}^{4} z_{2}^{14}-\frac{64}{75} z_{1}^{3} z_{2}^{15}\right] \oplus\left[z_{1}^{2} z_{2}^{17}\right]$.

(iii) Notice that

$$
\begin{aligned}
\operatorname{span}\left\{Q_{9,4} f, Q_{9,4} T f\right\} & =\operatorname{span}\left\{z_{1}^{9} z_{2}^{4}+z_{1}^{8} z_{2}^{5}, \frac{1}{3} z_{1}^{9} z_{2}^{4}+\frac{3}{8} z_{1}^{8} z_{2}^{5}\right\} \\
& =\operatorname{span}\left\{z_{1}^{9} z_{2}^{4}+z_{1}^{8} z_{2}^{5}, z_{1}^{9} z_{2}^{4}-\frac{27}{25} z_{1}^{8} z_{2}^{5}\right\}
\end{aligned}
$$

where $z_{1}^{9} z_{2}^{4}-\frac{27}{25} z_{1}^{8} z_{2}^{5} \perp Q_{9,4} f$. Then

$$
[f]=\left[z_{1}^{7} z_{2}^{7}\right] \oplus\left[z_{1}^{9} z_{2}^{4}+z_{1}^{8} z_{2}^{5}\right] \oplus\left[z_{1}^{9} z_{2}^{4}-\frac{27}{25} z_{1}^{8} z_{2}^{5}\right] .
$$


Remark 2.4. In Example 2.4, since $T^{*}\left(z_{1}^{9} z_{2}^{4}+z_{1}^{8} z_{2}^{5}\right)=\frac{1}{2} z_{1}^{4} z_{2}^{14}+\frac{4}{9} z_{1}^{3} z_{2}^{15}$ and $T^{*}\left(z_{1}^{9} z_{2}^{4}-\frac{27}{25} z_{1}^{8} z_{2}^{5}\right)=\frac{1}{2} z_{1}^{4} z_{2}^{14}-\frac{12}{25} z_{1}^{3} z_{2}^{15}$, conclusion (d) in Lemma 2.2 implies that $[f]=\left[z_{1}^{2} z_{2}^{17}\right] \oplus\left[\frac{1}{2} z_{1}^{4} z_{2}^{14}+\frac{4}{9} z_{1}^{3} z_{2}^{15}\right] \oplus\left[\frac{1}{2} z_{1}^{4} z_{2}^{14}-\frac{12}{25} z_{1}^{3} z_{2}^{15}\right]$.

Moreover, let $T=T_{z_{1}^{5} \bar{z}_{2}^{10}}$ and $g=z_{1}^{4} z_{2}^{14}+z_{1}^{9} z_{2}^{4}+z_{1}^{3} z_{2}^{15}$, then $[g]=[g+$ $\left.a z_{1}^{8} z_{2}^{5}\right]=\left[z_{1}^{4} z_{2}^{14}\right] \oplus\left[z_{1}^{3} z_{2}^{15}\right]$ for $a \neq \frac{9}{8}$. In fact, $\operatorname{span}\left\{P_{4,14}\left(g+a z_{1}^{8} z_{2}^{5}\right), P_{4,14} T^{*}(g+\right.$ $\left.\left.a z_{1}^{8} z_{2}^{5}\right)\right\}=\operatorname{span}\left\{z_{1}^{4} z_{2}^{14}, z_{1}^{3} z_{2}^{15}\right\}$, since $T^{*}\left(z_{1}^{9} z_{2}^{4}+a z_{1}^{8} z_{2}^{5}\right)$ and $z_{1}^{4} z_{2}^{14}+z_{1}^{3} z_{2}^{15}$ are linearly independent.

For the case that $a=\frac{9}{8}$, we have

$$
\begin{aligned}
{\left[g+\frac{9}{8} z_{1}^{8} z_{2}^{5}\right] } & =\operatorname{span}\left\{z_{1}^{4} z_{2}^{14}+z_{1}^{3} z_{2}^{15}, z_{1}^{9} z_{2}^{4}+\frac{9}{8} z_{1}^{8} z_{2}^{5}\right\} \\
& =\left[z_{1}^{4} z_{2}^{14}+z_{1}^{9} z_{2}^{4}\right]
\end{aligned}
$$

since $T^{*}\left(g+\frac{9}{8} z_{1}^{8} z_{2}^{5}\right)=\frac{1}{2} P_{4,14} g$.

Acknowledgments. The authors thank the reviewer very much for his helpful suggestions which led to the present version of this paper.

\section{References}

[1] H. Dan, and H. Huang, Multiplication operators defined by a class of polynomials on $L_{\alpha}^{2}\left(\mathbb{D}^{2}\right)$, Integral Equations Operator Theory 80 (2014), no. 4, 581-601.

[2] R. G. Douglas, M. Putinar, and K. Wang, Reducing subspaces for analytic multipliers of the Bergman space, J. Funct. Anal. 263 (2012), no. 6, 1744-1765.

[3] R. G. Douglas, S. Sun, and D. Zheng, Multiplication operators on the Bergman space via analytic continuation, Adv. Math. 226 (2011), no. 1, 541-583.

[4] K. Guo and H. Huang, On multiplication operators on the Bergman space: Similarity, unitary equivalence and reducing subspaces, J. Operator Theory 65 (2011), no. 2, 355378.

[5] _ Multiplication operators defined by covering maps on the Bergman space: the connection between operator theory and von Neumann algebras, J. Funct. Anal. 260 (2011), no. 4, 1219-1255.

[6] _ Geometric constructions of thin Blaschke products and reducing subspace problem, Proc. Lond. Math. Soc. 109 (2014), no. 4, 1050-1091.

[7] Multiplication Operators on the Bergman Space, Lecture Notes in Mathematics 2145, Springer-Verlag Berlin Heidelberg 2015.

[8] K. Guo, S. Sun, D. Zheng, and C. Zhong, Multiplication operators on the Bergman space via the Hardy space of the bidisk, J. Reine Angew. Math. 628 (2009), 129-168.

[9] J. Hu, S. Sun, X. Xu, and D. Yu, Reducing subspace of analytic Toeplitz operators on the Bergman space, Integral Equations Operator Theory 49 (2004), no. 3, 387-395.

[10] Y. Lu and X. Zhou, Invariant subspaces and reducing subspaces of weighted Bergman space over bidisk, J. Math. Soc. Japan 62 (2010), no. 3, 745-765.

[11] Y. Shi and Y. Lu, Reducing subspaces for Toeplitz operators on the polydisk, Bull. Korean Math. Soc. 50 (2013), no. 2, 687-696.

[12] M. Stessin and K. Zhu, Reducing subspaces of weighted shift operators, Proc. Amer. Math. Soc. 130 (2002), no. 9, 2631-2639.

[13] S. L. Sun and Y. Wang, Reducing subspaces of certain analytic Toeplitz operators on the Bergman space, Northeast. Math. J. 14 (1998), no. 2, 147-158.

[14] S. Sun, D. Zheng, and C. Zhong, Classification of reducing subspaces of a class of multiplication operators on the Bergman space via the Hardy space of the bidisk, Canad. J. Math. 62 (2010), no. 2, 415-438. 
[15] X. Wang, H. Dan, and H. Huang, Reducing subspaces of multiplication operators with the symbol $\alpha z^{k}+\beta \omega^{l}$ on $L_{a}^{2}\left(\mathbb{D}^{2}\right)$, Sci. China Math. 58 (2015), doi:10.1007/s11425-0154973-9.

[16] K. Zhu, Reducing subspaces for a class of multiplication operators, J. Lond. Math. Soc. 62 (2000), no. 2, 553-568.

Mohammed Albaseer

School of Mathematical Sciences

Dalian University of Technology

DALIAN 116024, P. R. ChINA

E-mail address: anm@mail.net.sa

YUfENG LU

School of Mathematical Sciences

Dalian University of Technology

Dalian 116024, P. R. China

E-mail address: lyfdlut@dlut.edu.cn

YANYUE SHI

School of Mathematical Sciences

OCEAN UNIVERSITY OF CHINA

QINGDAO 266100, P. R. CHINA

E-mail address: shiyanyue@163.com, shiyanyue@gmail.com 